\title{
Duplication of a well-conserved homeodomain-leucine zipper transcription factor gene in barley generates a copy with more specific functions
}

\author{
Shun Sakuma • Mohammad Pourkheirandish • \\ Takashi Matsumoto • Takato Koba • Takao Komatsuda
}

Received: 16 March 2009 /Revised: 7 July 2009 / Accepted: 3 August 2009 /Published online: 26 August 2009

(C) The Author(s) 2009. This article is published with open access at Springerlink.com

\begin{abstract}
Three spikelets are formed at each rachis node of the cultivated barley (Hordeum vulgare ssp. vulgare) spike. In two-rowed barley, the central one is fertile and the two lateral ones are sterile, whereas in the six-rowed type, all three are fertile. This characteristic is determined by the allelic constitution at the six-rowed spike 1 (vrs 1) locus on the long arm of chromosome $2 \mathrm{H}$, with the recessive allele (vrs l) being responsible for the six-rowed phenotype. The Vrsl (HvHoxl) gene encodes a homeodomain-leucine zipper (HD-Zip) transcription factor. Here, we show that the Vrs 1 gene evolved in the Poaceae via a duplication, with a second copy of the gene, HvHox2, present on the short arm of chromosome $2 \mathrm{H}$. Micro-collinearity and polypeptide sequences were both well conserved between HvHox 2 and its Poaceae orthologs, but Vrs1 is unique to the barley tribe. The Vrsl gene product lacks a motif which is conserved among the HvHox2 orthologs. A phylogenetic analysis demonstrated that $\operatorname{Vrs} 1$ and HvHox2 must have diverged after the separation of Brachypodium distachyon from the Pooideae and suggests that $\operatorname{Vrs} 1$ arose following
\end{abstract}

Electronic supplementary material The online version of this article (doi:10.1007/s10142-009-0134-y) contains supplementary material, which is available to authorized users.

S. Sakuma $\cdot$ M. Pourkheirandish $\cdot$ T. Matsumoto •

T. Komatsuda $(\square)$

National Institute of Agrobiological Sciences (NIAS),

Plant Genome Research Unit,

2-1-2 Kan-non-dai,

Tsukuba, Ibaraki 305-8602, Japan

e-mail: takao@affrc.go.jp

S. Sakuma $\cdot$ T. Koba

Graduate school of Horticulture, Chiba University, 648 Matsudo,

Matsudo, Chiba 271-8510, Japan the duplication of HvHox2, and acquired its new function during the evolution of the barley tribe. HvHox2 was expressed in all organs examined but $\operatorname{Vrs} 1$ was predominantly expressed in immature inflorescence.

Keywords Barley · Poaceae $\cdot$ Micro-collinearity · Gene duplication

$\begin{array}{ll}\text { Abbreviations } \\ \text { DNA } & \text { Deoxyribonucleic acid } \\ \text { cv } & \text { Cultivar } \\ \text { cDNA } & \text { Complementary DNA } \\ \text { BLAST } & \text { Basic local alignment search tool } \\ \text { NCBI } & \text { National Center for Biological Information } \\ \text { ESTs } & \text { Expressed sequence tags } \\ \text { PCR } & \text { Polymerase chain reaction } \\ \text { CAPS } & \text { Cleaved amplified polymorphic sequence } \\ \text { dCAPS } & \text { Derived CAPS } \\ \text { RNA } & \text { Ribonucleic acid } \\ \text { RT-PCR } & \text { Reverse transcription PCR }\end{array}$

\section{Introduction}

The grasses (Poaceae) form a monophyletic family of monocotyledonous plants which includes all the cereal crops, notably rice (Oryza sativa L.), maize (Zea mays L.), wheat (Triticum aestivum L.), barley (Hordeum vulgare L.), and sorghum (Sorghum bicolor L.). These cereals share a common ancestor from which they have diverged over a period of some 60 million years ago (Devos 2005); nevertheless, some synteny has been retained between them (Devos 2005; Gale and Devos 1998; Lu and Faris 2006). For example, rice chromosome 4 and 7 align well with 
chromosome 2 of barley and wheat (Chen et al. 2009; Devos 2005; Moore et al. 1995). With the complete rice genomic sequence to hand (International Rice Genome Sequencing Project 2005), it has become possible to demonstrate both where collinearity has been retained at the fine-scale level (Bennetzen and Ma 2003; Bossolini et al. 2007; Faris et al. 2008; Srinivasachary et al. 2007; Yan et al. 2003), and where it has collapsed as a result of inversions, deletions, duplications, and other intrachromosomal rearrangements (Ilic et al. 2003; La Rota and Sorrells 2004; Li and Gill 2002; Liu et al. 2006; Tarchini et al. 2000). Other full grass species genome sequencing project either completed or underway include those for sorghum (Paterson et al. 2009; Sasaki and Antonio 2009) and Brachypodium distachyon, a small genome, short growth cycle, self-fertile, model temperate grass(Ozdemir et al. 2008).

Inflorescence structure is one of the main determinants of grain yield in the cereals. The inflorescence can take the form of a panicle (rice, sorghum, and maize) or a spike (wheat, barley, and Brachypodium). Some evidence supports the notion that the spike has evolved from the panicle (Vegetti and Anton 1995). The barley spike carries a set of three spikelets at each rachis node. In "two-rowed" barley, the two lateral spikelets are reduced in size and sterile, but in the "six-rowed" type, all three spikelets are fertile. The six-rowed phenotype is genetically determined by homozygosity for the recessive allele (previously referred to as vrs 1 ) at the vrs 1 locus, which has been identified as a homeobox gene (HvHoxl) encoding a transcription factor containing a homeodomain (HD) with a leucine zipper motif (Zip; Komatsuda et al. 2007). HD-Zip proteins have been grouped into four families (Ariel et al. 2007), with the $V r s 1$ gene product (VRS1) belonging to the family I. Although HD can be found in all eukaryotic genomes, the HD-Zip family is restricted to the plant kingdom. The HDZip protein is dimerized by the Zip domain, and uses the HD to bind specifically to dyad-symmetrical DNA recognition sequences, based on the strict spatial relationship between HD and Zip (Sessa et al. 1993). VRS1 is thought to suppress the development of the lateral spikelets, since its expression was restricted to the lateral-spikelet primordia in the immature spikes (Komatsuda et al. 2007). The loss of Vrs 1 function resulted in the complete conversion of the rudimentary lateral spikelets of a two-rowed barley into fully developed fertile spikelets, just as in the six-rowed type.

Phylogenetic analysis demonstrated that the origin of the six-rowed phenotype was probably polyphyletic, both temporally and spatially, and occurred via a series of independent mutations at the $V r_{s} 1$ (Komatsuda et al. 2007). The higher seed set of the six-rowed type would have been readily selected during the domestication process (Harlan et al. 1973). Micro-collinearity between rice and barley is disrupted in the Vrs 1 region, but a Vrs 1 ortholog has been identified on rice chromosome 7 (Pourkheirandish et al. 2007). The barley EST (scsnp06322), mapping to the centromere region of chromosome $2 \mathrm{H}$, is homologous to rice Os07g0581000 (LOC Os07g39280), which co-locates with the rice Vrs1 ortholog Os07g0581700 (LOC_Os07g39320), (Pourkheirandish et al. 2007; Rostoks et al. 2005). This genomic location suggests the original site of $V r s 1$ to be the centromere region of chromosome $2 \mathrm{H}$ prior to the chromosomal rearrangement, which has been responsible for the local loss of synteny between rice and barley, but it is plausible that $\operatorname{Vrs} 1$ evolved as a 'copy' of an indispensable 'master' gene, which is still present in its ancestral location on chromosome $2 \mathrm{H}$ (Pourkheirandish et al. 2007). Neither the structure nor the function of $V r s 1$ orthologs in any of the other Poaceae members has been elucidated. The objective of this study was to compare the genomic organization of the regions containing a $V r s 1$ ortholog in a set of Poaceae species, as a means of inferring the refinement of the function of $\operatorname{Vrs} 1$ by gene duplication in the speciation of barley.

\section{Materials and methods}

\section{Plant materials}

The two-rowed barley cv. Kanto Nakate Gold (KNG, NIAS accession number JP 15436) and the six-rowed barley cv. Azumamugi (AZ, JP 17209; maintained in the Gene Bank, NIAS, Tsukuba, Japan) were intercrossed to allow the development of a population of $99 \mathrm{~F}_{12}$ recombinant inbred lines (RILs).The wild barley (H. vulgare ssp. spontaneum) strain OUH602 was obtained from Research Institute for Bioresources, Okayama University, Kurashiki, and used to generate a population of $186 \mathrm{~F}_{7}$ RILs from the cross OUH602 $\times$ KNG. A set of chromosome addition lines (CALs), in which six of barley cv. Betzes chromosomes $(2 \mathrm{H}-7 \mathrm{H})$ are present, in turn, in a background of bread wheat cv. Chinese Spring (Shepherd and Islam 1981) were kindly provided by Dr. A. K. M. R. Islam, Department of Plant Science, Waite Institute, University of Adelaide, Australia.

\section{Barley full-length cDNA library}

Seedling shoots and roots of cv. Haruna Nijo were used as a source of mRNAs to construct full-length cDNA libraries, following the methods described by Carninci et al. (1996). A sample of clones was end-sequenced (both 5' and 3'). A detailed description of this library and its construction will appear elsewhere. 
Searching for $V r s 1$ orthologs in Poaceae

Nucleotide-BLAST (BLASTN), protein-protein BLAST (BLASTP), and translated nucleotide-protein BLAST (TBLASTN) searches were made against the following sequence databases: barley, Barley Full-Length cDNA End Sequence Database of NIAS (unpublished); rice, Rice Annotation Project Database (http://rapdb.dna.affrc.go.jp/) and The Institute for Genomic Research (TIGR) Rice genome annotation (http://rice.plantbiology.msu.edu/); maize, MaizeSequence.org (http://www.maizesequence. org/index.html); sorghum, Department of Energy Joint Genome Institute (JGI) S. bicolor (http://genome.jgi-psf. org/Sorbi1/Sorbi1.download.html); B. distachyon, BRACHYPODIUM.ORG (http://www.brachypodium.org/); wheat, TIGR Wheat Genome database (http://www.tigr. org/tdb/e2k1/tae1/), and NCBI (http://blast.ncbi.nlm.nih. gov/Blast.cgi).

Phylogenetic and peptide motif analysis

Sequence data were aligned using ClustalW2 software (http://www.ebi.ac.uk/Tools/clustalw2/). A phylogenetic tree was constructed by the neighbor-joining method, using PAUP 4.0b10 software (Sinauer, Sunderland, Massachusetts) employing 100 bootstrap replicates. Insertion/deletion characters were not included. Peptide motifs were analyzed using the Surveyed conserved motif ALignment diagram and the Associating Dendrogram (SALAD) database (http://salad.dna.affrc.go.jp/salad/en/; Mihara et al. 2008). A graphical display of motif composition was constructed using Interactive SALAD analysis.

\section{Barley ESTs data}

Barley ESTs giving the best match to rice genes on chromosome 7 were selected from the Gramene database (http://www.gramene.org/Oryza_sativa_japonica/index. html). The copy number of each EST was investigated using Plant Repeat Database at Michigan State University (http://plantrepeats.plantbiology.msu.edu/index.html). Exon-intron junctions were assumed to be conserved between rice and barley, and were extracted from NCBI BLAST 2 SEQUENCES (http://blast.ncbi.nlm.nih.gov/bl2seq/ wblast2.cgi).

\section{PCR amplification}

Plant genomic DNA was extracted as described by Komatsuda et al. (1998). PCR primers were designed from the predicted exon regions with Oligo5 software (W. Rychlick, National Bioscience, Plymouth, MN, USA) and synthesized commercially (BEX, Tokyo, Japan) (Supple- mentary Table 2). PCR amplification was carried out in $10 \mu 1$ reactions containing $0.25 \mathrm{U}$ ExTaq polymerase (Takara, Tokyo, Japan), 1× ExTaq polymerase buffer, $0.3 \mu \mathrm{M}$ of each primer, $200 \mu \mathrm{M}$ dNTP, $2 \mathrm{mM} \mathrm{MgCl}_{2}$, $0-5 \% \quad v / v$ dimethyl sulphoxide (DMSO), and $20 \mathrm{ng}$ genomic DNA. Each PCR was cycled through a denaturation step $\left(94^{\circ} \mathrm{C} / 5 \mathrm{~min}\right)$, followed by 30 cycles of $94^{\circ} \mathrm{C} / 30 \mathrm{~s}$, $55-65^{\circ} \mathrm{C}$ (primer-dependent) $/ 30$ s, $72^{\circ} \mathrm{C} / 30-90$ s with a final incubation of $72^{\circ} \mathrm{C} / 7 \mathrm{~min}$. Amplicons were electrophoresed through either agarose (Agarose ME, Iwai Kagaku, Tokyo, Japan) or a MetaPhor agarose (Cambrex Bio Science Rockland Inc., Rockland, MA, USA) gels, depending on their size, and were visualized by ethidium bromide staining.

Development of CAPS and dCAPS markers

PCR products were purified using the QIAquick PCR purification Kit (Qiagen, Germantown, MD, USA) and subjected to cycle sequencing using a Big Dye Terminator Kit (Applied Biosystem, Foster, CA, USA). Sequencing reactions were purified by Agencourt CleanSEQ (Beckman, Beverly, MA, USA), and analyzed with an ABI prism 3130 genetic analyzer (Applied Biosystem). Sequence data were aligned by Sequencher DNA Sequencing Software (HitachiSoft, Yokohama, Japan). Polymorphic restriction sites were identified via the Restriction Maps option of Molecular Toolkit (http://arbl.cvmbs.colostate.edu/molkit/mapper/) or with dCAPS Finder 2.0 (http://helix.wustl.edu/dcaps/dcaps. $\mathrm{html})$. PCR products were digested at the recommended temperature for $2-3 \mathrm{~h}$ in reactions of $15 \mu \mathrm{l}$ of reaction mixture containing $10 \mu \mathrm{l}$ PCR products, $1 \times$ reaction buffer, and $1 \mathrm{U}$ restriction enzyme.

\section{Genetic mapping}

The AZ $\times$ KNG and OUH602 $\times$ KNG RILs population were genotyped using polymorphic CAPS and dCAPS markers, and MAPMAKER/EXP ver.3.0 (Lander et al. 1987) was used to integrate the resulting loci into the linkage map constructed by Komatsuda and Tanno (2004).

RNA extraction and RT-PCR assay

Total RNA was extracted from various tissues by using TRIzol (Invitrogen, Carlsbad, CA). A first-strand cDNA was synthesized with SuperScript II (Invitrogen). The first-strand cDNA was used as a template, and amplification was performed for 30 or 40 PCR cycles $\left(1 \mathrm{~min}\right.$ at $94^{\circ} \mathrm{C}, 30 \mathrm{~s}$ at $60 / 65^{\circ} \mathrm{C}, 30 \mathrm{~s}$ at $72^{\circ} \mathrm{C}$ ) followed by $7 \mathrm{~min}$ at $72^{\circ} \mathrm{C}$. The primers for $\mathrm{HvHox} 2$ were 5'-GCGTGGTCGAGTGGTTTAGCCTGT-3' (sense) and 5'-GAGAGCTACCGGTACTACACTTGC-3' (anti- 


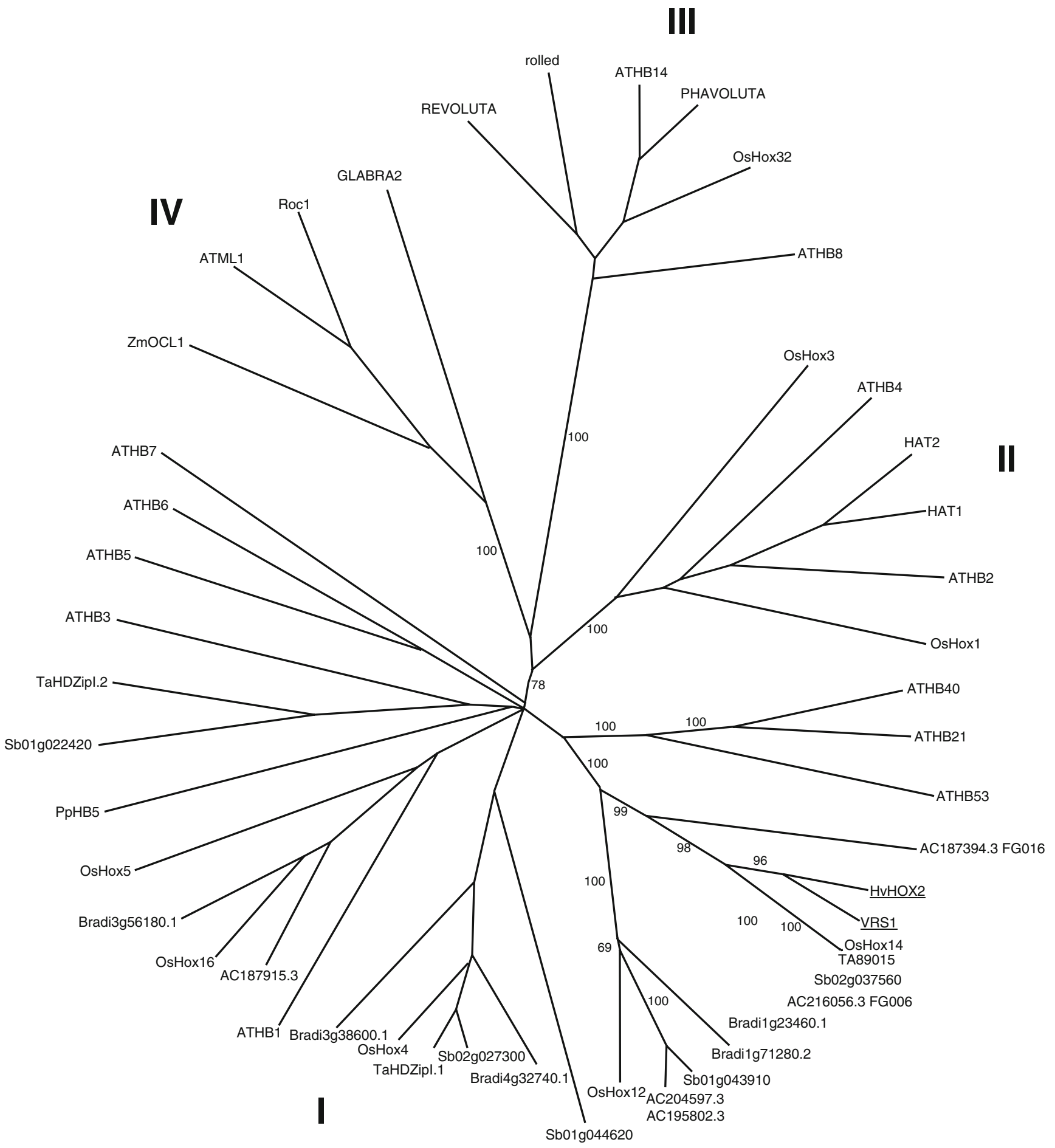

0.1 changes

Fig. 1 Phylogenetic analysis of the predicted peptide sequences of the Vrs 1 homologues among the Poaceae and HD-Zip family genes in $A$. thaliana. The phylogenetic tree was constructed by the neighbor- joining method using PAUP 4.0b10. Local bootstrap values after 100 replicates are indicated near the branching points 
sense). Vrsl primers were 5'-GGTTTTTAGCATGAATTA GAGTTTA-3' (sense) and 5'-TATACAGGCTAAAAACCA AAGATTA-3' (antisense). Actin primers were 5'GTCCTTTTCCAGCCATCTTTC-3' (sense) and 5'-CAA GAATCGACCCTCCAATCC-3' (antisense). RT-PCR assay was performed at least twice for each sample.

\section{Results}

Identification of a $V r s 1$ paralog in barley

The best hit ( $E$-value, e-110) from a BLASTN search based on the Vrs 1 cDNA sequence (accession no. AB259783) as a

Bradilg23460.1
HvHOX2
VRS1
TA89015
Os07g0581700
Sb02g037560
AC216056.3_FG006
AC187394.3_FG016

Bradilg23460.1 HvHOX2

VRS1

TA89015

Os $07 g 0581700$

$\mathrm{Sb} 02 \mathrm{~g} 037560$

AC216056.3_FG006

AC187394.3_FG016

$10 \quad 20$

30

40

50

60

MD-----KQ ELFASSYMGT S--FLGANG- TAAVQGERPR ARRRRRRAAA ARCEDVDGGL MD--.--KQ HLFGCSYVDA P--FFAATG- TAQGE-SRPR ARRRRRRAAR CGGGDGDGVE MD------KH QLFDSSNVDT T--FFAANG- TAQGDTSKQR ARRRRRRSAR CGGGDGDGGE MDRYGEKQQQ QQMFASYVDA S--LLAASG- EVQGE--RPR ARRRRRRGAR CVGGGGGGGE MDRYGEKQQQ QQMFASYVDA S--LLAASG- EVQGE--RPR ARRRRRRGAR CVGGGGGGGE MEE-----YD GLFPSAYVDS SSSLLVPNG- TAQGE--RPR ARRRRRRAPR CGGGG----D MEQ----YYD GLFPSAYADS SSSLLMPNGS KAQGE--RPR ARRRRRRAAW CGGG-----E MEQ----YD GLFPPAYVDS SSSLLLVPNA NGTAQEERPR ARRRRRRAAR CGGG---GGE

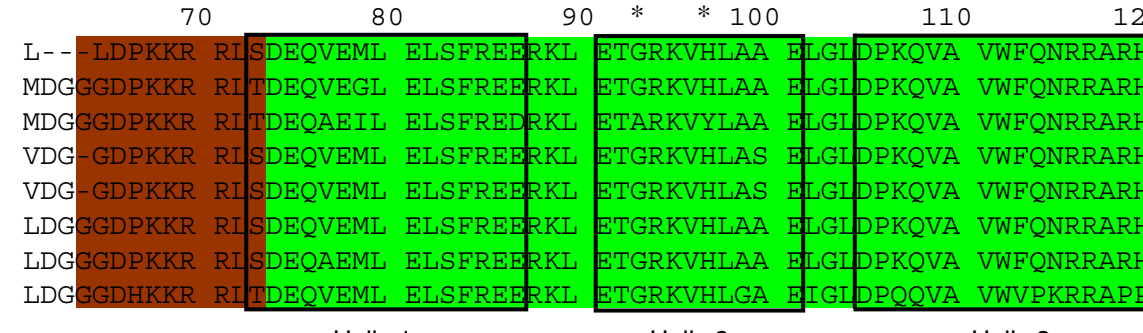

120

Bradilg23460.1
HvHOX2
VRS1
TA89015
Os07g0581700
Sb02g037560
AC216056.3_FG006
AC187394.3_FG016

Bradilg23460.1 HvHOX2

VRS1

TA89015

Os $07 \mathrm{~g} 0581700$

$\mathrm{Sb} 02 \mathrm{~g} 037560$

AC216056.3 FG006

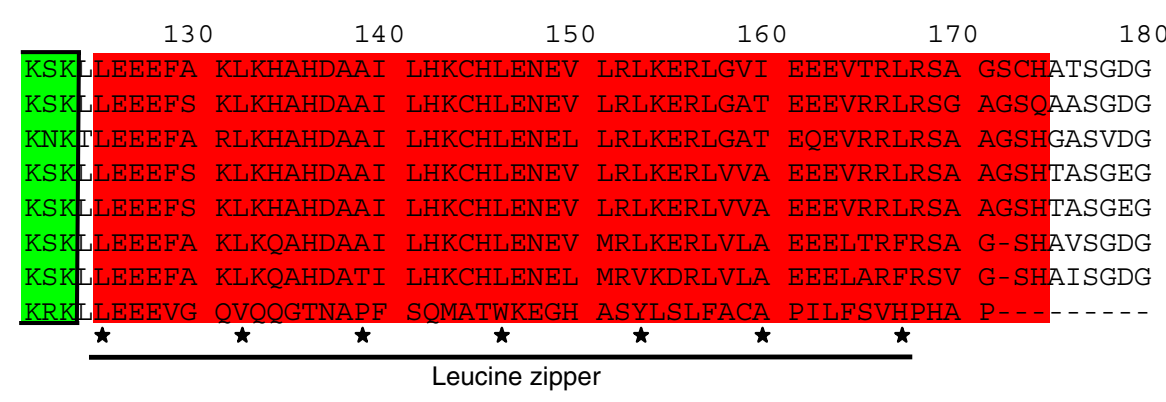
190
200
210
220
230

240

---AGHH-- - ---GSPSSS FSTGTCHHQQ QPGFTGG--- ADVMLGNDDL MMYVPDAEYG GDAAGAVG- - -LCGGSPSSS FSTGTC--QQ HPGFSG--- ADVLGPDDDL MMCVPEYGGGHAAGAVG-- -VCGGSPSSS FSTGTC--QQ QPGFSG---- ADVLGRDDDL MMCVPEWFLA GDIMGLGGSG ACVAGSPSSS FSTGTC---Q PPSFGGG--- -DHLGDDD-- LVYVPEYGGGDIMGLGGSG ACVAGSPSSS FSTGTC---Q PPSFGGGGGG GDHLGDDD-- LVYVPEYGGGD-IMGRA-- -VCSGSPSSS FSTGTC---H QPGVDVG--- GGDHLGDDDQ LLYVPDYAYGDAMMGRA-- -VCSGSPSSS FSTGTC---Q QP--.--.- ----GDDD- LLYFPDYAY-

AC187394.3_FG016
250 HvHOX2

VRS1

TA89015

Os $07 \mathrm{~g} 0581700$

$\mathrm{Sb} 02 \mathrm{~g} 037560$

AC216056.3 FG006

AC187394.3_FG016

Motif 1

Motif 2
Bradilg23460.1
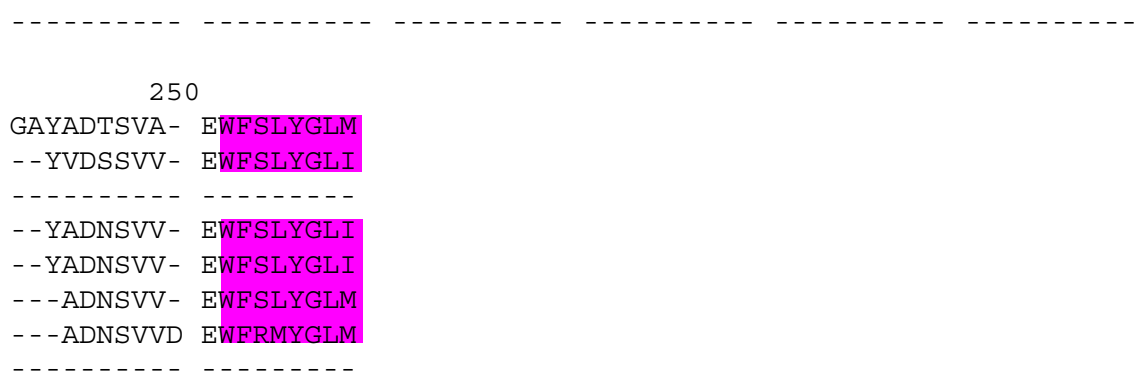

Motif $7 \square$ Motif 8

Fig. 2 Comparison between the deduced peptide sequences of VRS1, HvHOX2 and its Poaceae orthologs. The three homeodomain helical sequences are shown boxed, and a leucine zipper is indicated by staring and underlining. Hyphens indicate a gap introduced to facilitate alignment. Colored boxes show the motifs. Asterisks show the position of Ala 84 (A) and Tyr 88 (Y) in VRS1 
query was the barley full-length cDNA clone Hv2074A10 (accession no. AB490233) extracted from the seedling shoots and root of cv. Haruna Nijo (NIAS barley database). Four lesser hits $(E$-values $<\mathrm{e}-10)$ were also identified from the same library. The search using $V r s 1$ HD-Zip coding region as the query sequence hit three entries $(E$-values $<$ - 10$)$ in the same database. Vrsl and Hv2074A10 shared an identical exon/intron structure (Supplementary Fig. 1). The second and third exons of both genes contained homeodomainleucine zipper motif. As a result, the Hv2074A10 was named "HvHox2". Alignment of the Vrs1 and HvHox2 cDNA sequences identified a 300-bp insertion in $\operatorname{Vrs} 1$ and a $44 \mathrm{bp}$ insertion in the third exon of HvHox2 (Supplementary Fig. 2). Neither the 300 nor the $44 \mathrm{bp}$ sequences were homologous to any accession in the public domain. The $V r s 1$ insertion resulted in the generation of a stop codon, producing a polypeptide 14 residues shorter than the HvHox2 product (Supplementary Fig. 3). The deduced polypeptide sequences of the HvHox2 (HvHOX2, 236 aa) and Vrs1 (VRS1, 222 aa) shared $88 \%$ identity in the HD-Zip domain and $84 \%$ identity in the whole protein.

\section{Identification of $\operatorname{Vrs} 1$ homologues in the Poaceae}

The $V r s 1$ cDNA sequence was used to query each species database in turn. Vrsl homologues were present in rice (Os07g0581700, Oshox14), B. distachyon (Bradi1g23460.1), sorghum (Sb02g03750), and maize (AC216056.2_FG006 and AC187394.3_FG016). The presence of two (rather than just one) Vrs 1 homologues in maize probably reflects the cryptic tetraploidy of the maize genome (Paterson et al. 2004; Swigonova et al. 2004; Wei et al. 2007). BLASTN search using HvHox2 as the query sequence produced the same hits. All the Vrs 1 homologues contained an HD-Zip motif (Supplementary Fig. 3).

\section{Phylogenetic analysis of VRS1 homologues}

Polypeptide sequences matching that of VRS1 were selected by BLASTP or TBLASTN search against the wheat, $B$. distachyon, rice, sorghum, and maize databases (Supplementary Table 1). The top five sequences with significant similarity to VRS1 were selected from each taxon to form a phylogenetic tree. Arabidopsis thaliana HD-Zip family (classes I-IV) proteins were included to infer the class to which the Poaceae proteins belonged. The resulting neighbor-joining tree showed a clear separation of the four families of HD-Zip families, with each clad being strongly supported by bootstrap analysis (Fig. 1). All the VRS1 homologues clustered within the HD-Zip I family (along with A. thaliana ATHB40, ATHB21, and ATHB53), suggesting that these sequences are orthologous. The high degree of similarity between VRS1 and HvHOX2 (boot- strap support level 88\%) suggests that they became separated from one another after the separation of barley (Fig. 1). The sister clad, containing the rice, B. distachyon, wheat, sorghum and maize sequences, was also highly supported (100\%). The peptide motifs 1 and 7 within VRS1 form the homeodomain and motif 2 forms the leucine zipper (Fig. 2). Motif 3, 4, and 6 were highly conserved across all the orthologs (Supplementary Fig. 4) - their function is unknown. Motif 8 , in the C-terminal region of the gene, was highly conserved across all the non-barley orthologs and HvHOX2, but not in VRS1 (Fig. 2). Thus, we concluded that $H v H o x 2$, rather than $\operatorname{Vrs} 1$, is probably the original representative of the gene in barley.

Genetic mapping of HvHox2 in barley

HvHox2 mapped $27.1 \mathrm{cM}$ from $v r s 1$ on chromosome $2 \mathrm{H}$ (OUH602 $\times$ KNG; Fig. 3). A more saturated map of the HvHox 2 region was obtained by including the loci corresponding to 24 barley ESTs with homology to rice genes in the chromosome 7 containing the rice HvHox2 ortholog Oshox14 (syn. Os07g0581700). PCR primers targeting the predicted exon regions generated a singlePCR product for 20 of these 24 ESTs (Supplementary Table 2). CALs analysis assigned eight of 20 to chromosome $2 \mathrm{H}$, with the remaining 12 non-assigned, because the wheat and barley amplicons co-migrated on the gels. The OUH602 and KNG amplicon sequence differed for ten of the EST sequences, but in the contrast between AZ and $\mathrm{KNG}$, only one amplicon sequence was polymorphic. Based on the OUH602 $\times$ KNG RILs, five of the loci cosegregated with $H v H o x 2$, mapping to a location $0.6 \mathrm{cM}$ proximal to the AV924741 and $3.7 \mathrm{cM}$ proximal to $A B G 602$ on the short arm of chromosome $2 \mathrm{H}$ (Fig. 3).

Micro-collinearity in the Poaceae

The outcome of a study of fine-scale micro-collinearity in the HvHox2 homologue region between rice chromosome 7 and B. distachyon Bd. 1 is shown in Supplementary Table 3.

Fig. 3 Micro-collinearity among the Poaceae in the Vrs1 and HvHox2 regions. The maps of Brachypodium, rice, sorghum, and maize are physical, and barley genetical. Dotted lines connect barley markers with their Poaceae orthologs. Broken lines connect HvHox2 with their Poaceae orthologs. In the barley genetic maps, OUH602 $\times$ KNG (left), $\mathrm{AZ} \times \mathrm{KNG}($ right), markers shown in italics are PCR marked loci derived from RFLPs and AFLPs, while other markers are ESTderived. Markers with 'BC' denote barley EST clusters: BC15586 (CB872532 and CA025026), BC11013 (BF064588 and BJ453556), $\mathrm{BC} 12348$ (AJ468022 and $\mathrm{CB} 881790$ ), BC12063 (BU973565 and BU987455), BC14030 (CA005338 and CA019164), BC17525 (AJ486478 and BQ664265), BC09675 (AV920420and BM100120) and BC02453 (BM816591; after Pourkheirandish et al. 2007). Scale bar shows $1 \mathrm{Mb}$ 
Maize

Chr. $2 \mathrm{~L} \quad$ Chr. 7

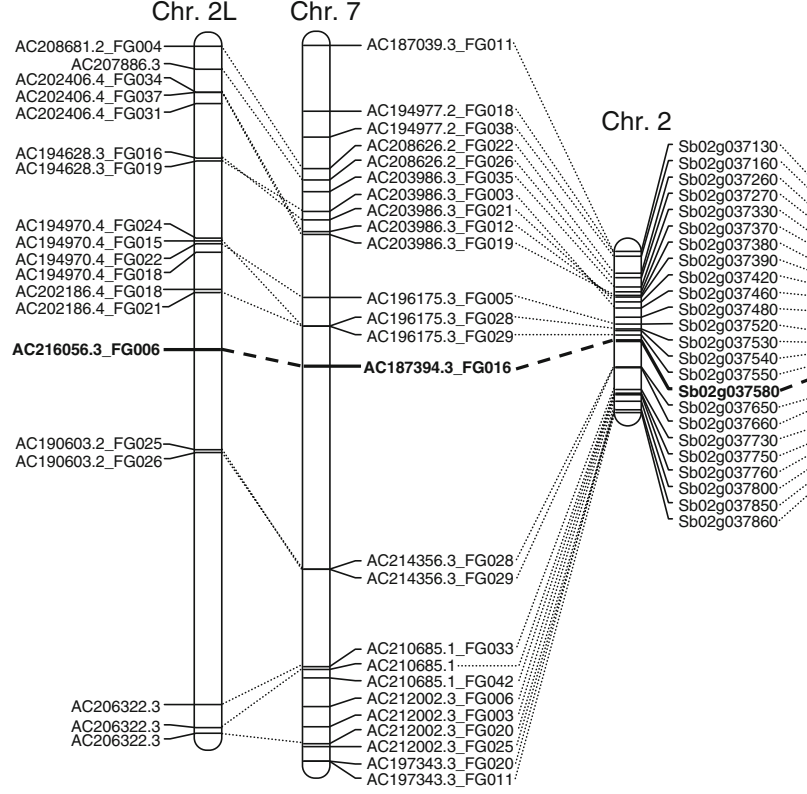

$$
\text { Chr. 2S }
$$

AC189043.3 231405.1

AC189043.3 FG027

AC189043.3_FG021

$\mathrm{AC} 189043.3 \mathrm{FG} 005$
AC1 94139.3

AC194139.3 FG016

AC206615.3_FG009

AC205850.4_FG010

AC213606.2_FG014

AC213606.2_FG003 -1 :

AC204221.3_FG007

AC187339.3_FG006

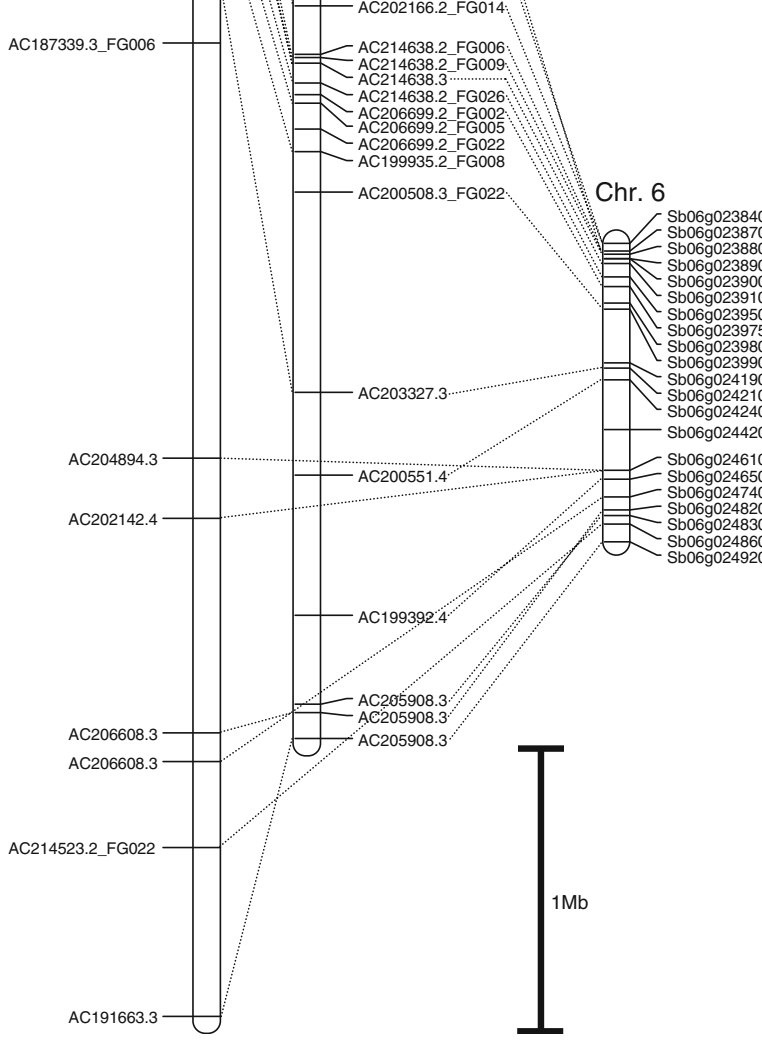

Rice

Chr. 7

Os07g0573400

Os07g0574800

Os079575100
Os0790575800
Os07057000

Os07g0576000
Os070577300

Os0795777300

Os0790577700
Os07g0577900

Os 0790578600
Os07g0581000

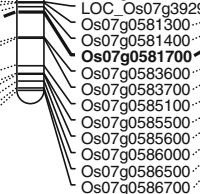

Brachypodium

Barley

Chr. $2 \mathrm{H}$ genetic

Bd.

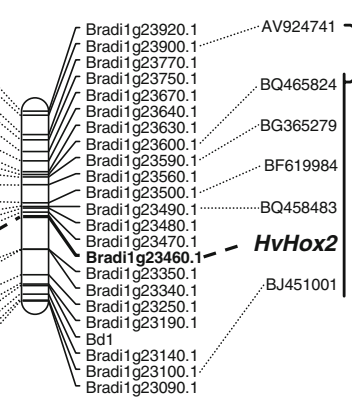

ए $A B G 60$ 
A series of BLASTN searches based on rice cDNA queries showed that both gene contents and orthologs order was well conserved between these two species (Fig. 3). Similarly, micro-collinearity in the HvHox2 region was conserved between rice chromosome 7 , sorghum chromosome 6 , and maize chromosomes $2 \mathrm{~S}$ and 10 (Fig. 3). Micro-collinearity was also conserved in the $V r s 1$ region (rice chromosome 4, $B$. distachyon $\mathrm{Bd}$. 5, sorghum chromosome 2 and maize chromosome $2 \mathrm{~L}$ and 7-see Fig. 3). However, there were no Vrs1 orthologs present in this region in any of these species.

Expression analysis of $H v H o x 2$ and $V r s 1$

RT-PCR analysis showed that HvHox2 was expressed in all four organs tested (leaves, shoots and roots of the seedlings, and immature inflorescences in $5 \mathrm{~mm}$ long; Fig. 4). Vrs 1 was strongly expressed in the immature inflorescences but slightly or not expressed in the other organs. There was no difference of gene expression pattern between $\mathrm{AZ}$ and KNG.

\section{Discussion}

\section{A duplication of $H v H o x 2$ gave rise to $\operatorname{Vrs} 1$}

Micro-collinearity can be disrupted by inversion, tandem duplication, indel formation, or transposition (Devos 2005). Two alternative hypotheses have been proposed to account for the loss of micro-collinearity between barley and rice in the Vrs1 region: (1) the chromosomal segment containing Vrs1 was transposed from the short arm to the long arm within chromosome $2 \mathrm{H}$; or (2) Vrs 1 evolved from a copy of an indispensable master gene, which is still present in its ancestral location on chromosome $2 \mathrm{H}$ (Pourkheirandish et al. 2007). The results of the present study are supportive of the latter hypothesis, since the phylogenetic analysis suggested that HvHox2 and $V r s 1$ are paralog (Fig. 1), while the comparative genetic mapping showed that the ancestral copy is HvHox2, not $\operatorname{Vrs} 1$ (Fig. 3). The duplication of $H v H o x 2$ must have occurred after the separation of $B$. distachyon from the Pooideae (Fig. 1), an event which has been timed at $\sim 35-40$ million years ago (Catalan and Olmstead 2000). It seems probable that HvHox2 and Vrs 1 diverged after the split between Triticum and Hordeum, because no $\operatorname{Vrs} 1$ homologue is represented in the wheat EST database, whereas a wheat HvHox 2 ortholog has been identified. Vrsl is present in H. vulgare ssp. spontaneum (Komatsuda et al. 2007; this study) and H. bulbosum (M. Pourkheirandish et al. unpublished) but until its distribution among Hordeum spp. more generally has been established, it is not possible to estimate how early during the evolution of this tribe the duplication event occurred. Although it is not possible to exclude the possibility of the 'duplicated' $V r s 1$ pseudogene formation in process of the speciation in the other taxa of Poaceae, a BLAST search did not detect any pseudogenes located in the Vrs 1 region in other species (Fig. 3, Supplementary Table 4).

The HvHox2 sequence is highly conserved among the Poaceae

The HD-Zip I genes share intron and exon distribution (Ariel et al. 2007), and HvHox2 and its Poaceae orthologs have a similar genomic structure, including HD-Zip motif and peptide motifs (Fig. 2). The expression of HD-Zip I genes is regulated by drought, cold, and osmotic stresses, and by various hormones (Dezar et al. 2005; Himmelbach et al. 2002; Olsson et al. 2004; Rueda et al. 2005). Treatment of $A$. thaliana plants with either abscisic acid or salt increased the transcription of ATHB21, -40, -53 genes which are phylogenetically closest to the HvHox2 (Fig. 1). Most HD-Zip genes (including the three above) are expressed (in seedlings, roots, leaves, stems, and flowers), but a few show organ-specific patterns of expression (Agalou et al. 2008; Henriksson et al. 2005). HvHox2 was expressed in the leaves, shoots, roots, and immature inflorescences, while Vrsl was expressed only in the immature inflorescences (Fig. 4). In situ hybridization showed that $\operatorname{Vrs} 1$ expression was very much localized to the lateral-spikelet primordia in spikes at the triple-mound stage and glum-primordium stages (Komatsuda et al. 2007), with zero detectable expression in young leaves (Fig. 4). While HvHox2 and $V r s 1$ may regulate a wholly or partially overlapping set of downstream genes, their biological functions may have been differentiated by their expression profile. On the basis of polypeptide sequences, it seems likely that the molecular role of HvHox2 and its Poaceae orthologs is identical. Within barley itself, the genome sequence of HvHox2 (accession no. AB490234) in the tworowed type (KNG and Haruna Nijo) is the same as that in

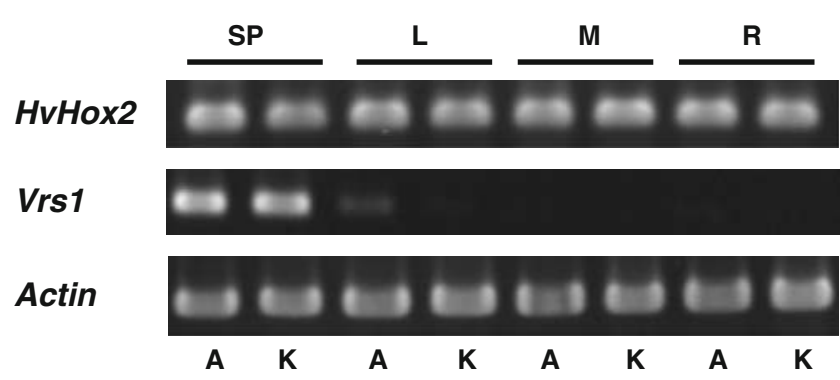

Fig. 4 Expression analysis of HvHox2 and $V r s 1$ by RT-PCR. SP, immature inflorescence (5 $\mathrm{mm}$ in length); $L$ leaves of seedling, $M$ coleoptile node segment of seedling, $R$ roots of seedling. $A$ six-rowed barley cv. Azumamugi, $K$ two-rowed barley cv. Kanto Nakate Gold 
the six-rowed type (AZ and Morex) (Supplementary Fig. 1), whereas the $\operatorname{Vrs} 1$ sequence is remarkably variable among these same cultivars (Komatsuda et al. 2007). The lack of a Oshox 14 mutant in the Tos 17 insertion mutant collection (Hirochika 2001) does not contradict with the notion that HvHox2 is essential for plant growth and development in the cereals. In contrast, the loss of $\mathrm{Vrs} 1$ function both in natural variants, and in many induced mutants (including the total deletion of the gene) indicates that $V r s 1$ is non-essential for plant growth and development (Komatsuda et al. 2007).

\section{Neo-functionalization of $\operatorname{Vrs} 1$}

Gene duplication is closely associated with creation of novel gene functions (Force et al. 1999). Most paralogs are lost after few million years, but those which either gain a new function ("neo-functionalization") or cover old function ("sub-functionalization") survive. In rice, the two C-class MADS box genes OSMADS3 and OSMADS58, which were generated by a gene duplication, have been partially sub-functionalized (Yamaguchi et al. 2006). While $H v H o x 2$ probably has retained its ancestral functions, $V r s 1$ may have acquired the role of suppressing the lateralspikelet development (Fig. 4). Vrs1 expression was localized to the lateral-spikelet primordia (Komatsuda et al. 2007). Presumably, this phenotype is associated with a selective advantage in nature (Pourkheirandish and Komatsuda 2007). It is unclear, however, whether the recruitment of $V r s 1$ to its present function occurred before or after the gain of the triplet in Hordeum.

HD-Zip proteins form dimers that recognize the pseudopalindromic DNA sequence (Chan et al. 1998; Palena et al. 1999, 2001). Two residues in helix II, and one in the loop between helix I and helix II make contact with the target DNA (Tron et al. 2004), and these contacts are critical for aligning the recognition helix correctly. In the VRS1 protein, the glycine 84 and histidine 88 residues present in the helix II region of HvHOX2 and its Poaceae orthologs have been mutated to alanine 84 and tyrosine 88 , respectively (Fig. 2). One, or both of these alterations may change either binding affinity or the target DNA sequence. However, if HvHOX2 and VRS1 still share the same target DNA sequence and retain the same level of affinity, it is possible that VRS1 competes with HvHOX2 to bind to cis-elements of downstream genes. Since Vrsl is expressed only in the lateralspikelet primordia, VRS1 would tend to out-compete HvHOX2 for binding in the lateral-spikelet primordia. The 300-bp insertion into the $V r s 1$ sequence both introduced a new stop codon (Supplementary Fig. 3) and removed a conserved peptide in motif 8 in the $\mathrm{C}$-terminal region (Fig. 2). The role of the motif 8 is not annotated in the protein family (Pfam) database (http://pfam.sanger.ac.uk/), but its loss may be sufficient to differentiate the functions of the $V r s 1$ and HvHox 2 gene products. Transcriptional coactivators enhance transcription by interacting with both general and gene-specific transcription factors (Zanetti et al. 2004). Thus, motif 8 in HvHOX 2 could interact with certain classes of transcriptional co-activators, and then act as an activator for the transcription of downstream genes, while VRS1 would not be able to replicate this interaction as it lacks motif 8 . The formation of HvHOX2/VRS1 heterodimers may reduce the concentration of HvHOX2 homodimer in the cell. None of the above three hypotheses are mutually exclusive, so may be combined to explain the function of Vrsl.

Acknowledgments We thank N. Wang, M. Sameri, G. Chen, M. Mihara, H. Sassa and S. Kikuchi for their help and advice. We also thank R. Koebner for linguistic assistance in the preparation of this manuscript. This research was financially supported by the Ministry of Agriculture, Forestry, and Fisheries of Japan (Genomics for Agricultural Innovation grant no. TRC1004).

Open Access This article is distributed under the terms of the Creative Commons Attribution Noncommercial License which permits any noncommercial use, distribution, and reproduction in any medium, provided the original author(s) and source are credited.

\section{Reference}

Agalou A, Purwantomo S, Overnas E, Johannesson H, Zhu X, Estiati A, de Kam RJ, Engstrom P, Slamet-Loedin IH, Zhu Z, Wang M, Xiong L, Meijer AH, Ouwerkerk PB (2008) A genome-wide survey of HD-Zip genes in rice and analysis of droughtresponsive family members. Plant Mol Biol 66:87-103

Ariel FD, Manavella PA, Dezar CA, Chan RL (2007) The true story of the HD-Zip family. Trends Plant Sci 12:419-426

Bennetzen JL, Ma J (2003) The genetic colinearity of rice and other cereals on the basis of genomic sequence analysis. Curr Opin Plant Biol 6:128-133

Bossolini E, Wicker T, Knobel PA, Keller B (2007) Comparison of orthologous loci from small grass genomes Brachypodium and rice: implications for wheat genomics and grass genome annotation. Plant J 49:704-717

Carninci P, Kvam C, Kitamura A, Ohsumi T, Okazaki Y, Itoh M, Kamiya M, Shibata K, Sasaki N, Izawa M, Muramatsu $M$, Hayashizaki Y, Schneider C (1996) High-efficiency full-length cDNA cloning by biotinylated CAP trapper. Genomics 37:327336

Catalan P, Olmstead RG (2000) Phylogenetic reconstruction of the genusBrachypodium P. Beauv. (Poaceae) from combined sequences of chloroplast $n d h \mathrm{~F}$ gene and nuclear ITS. Plant Syst Evol 220:1-19

Chan RL, Gago GM, Palena CM, Gonzalez DH (1998) Homeoboxes in plant development. Biochim Biophys Acta 1442:1-19

Chen A, Brule-Babel A, Baumann U, Collins NC (2009) Structurefunction analysis of the barley genome: the gene-rich region of chromosome 2HL. Funct Integr Genomics 9:67-79

Devos KM (2005) Updating the 'crop circle'. Curr Opin Plant Biol 8:155-162

Dezar CA, Gago GM, Gonzalez DH, Chan RL (2005) Hahb-4, a sunflower homeobox-leucine zipper gene, is a developmental 
regulator and confers drought tolerance to Arabidopsis thaliana plants. Transgenic Res 14:429-440

Faris JD, Zhang Z, Fellers JP, Gill BS (2008) Micro-colinearity between rice, Brachypodium, and Triticum monococcum at the wheat domestication locus $Q$. Funct Integr Genomics 8:149-164

Force A, Lynch M, Pickett FB, Amores A, Yan YL, Postlethwait J (1999) Preservation of duplicate genes by complementary, degenerative mutations. Genetics 151:1531-1545

Gale MD, Devos KM (1998) Comparative genetics in the grasses. Proc Natl Acad Sci USA 95:1971-1974

Harlan JR, JMJd W, Price EG (1973) Comparative Evolution of Cereals. Evolution 27:311-325

Henriksson E, Olsson AS, Johannesson H, Johansson H, Hanson J, Engstrom P, Soderman E (2005) Homeodomain leucine zipper class I genes in Arabidopsis. Expression patterns and phylogenetic relationships. Plant Physiol 139:509-518

Himmelbach A, Hoffmann T, Leube M, Hohener B, Grill E (2002) Homeodomain protein ATHB6 is a target of the protein phosphatase ABI1 and regulates hormone responses in Arabidopsis. EMBO J 21:3029-3038

Hirochika H (2001) Contribution of the Tos 17 retrotransposon to rice functional genomics. Curr Opin Plant Biol 4:118-122

Ilic K, SanMiguel PJ, Bennetzen JL (2003) A complex history of rearrangement in an orthologous region of the maize, sorghum, and rice genomes. Proc Natl Acad Sci USA 100:12265-12270

International Rice Genome Sequencing Project (2005) The map-based sequence of the rice genome. Nature 436:793-800

Komatsuda T, Tanno K (2004) Comparative high resolution map of the six-rowed spike locus 1 (vrs 1 ) in several populations of barley, Hordeum vulgare L. Hereditas 141:68-73

Komatsuda T, Nakamura I, Takaiwa F, Oka S (1998) Development of STS markers closely linked to the vrs 1 locus in barley, Hordeum vulgare. Genome 41:680-685

Komatsuda T, Pourkheirandish M, He C, Azhaguvel P, Kanamori H, Perovic D, Stein N, Graner A, Wicker T, Tagiri A, Lundqvist U, Fujimura T, Matsuoka M, Matsumoto T, Yano M (2007) Sixrowed barley originated from a mutation in a homeodomainleucine zipper I-class homeobox gene. Proc Natl Acad Sci USA 104:1424-1429

La Rota M, Sorrells ME (2004) Comparative DNA sequence analysis of mapped wheat ESTs reveals the complexity of genome relationships between rice and wheat. Funct Integr Genomics 4:34-46

Lander ES, Green P, Abrahamson J, Barlow A, Daly MJ, Lincoln SE, Newburg L (1987) MAPMAKER: an interactive computer package for constructing primary genetic linkage maps of experimental and natural populations. Genomics 1:174-181

Li W, Gill BS (2002) The colinearity of the Sh2/A1 orthologous region in rice, sorghum and maize is interrupted and accompanied by genome expansion in the triticeae. Genetics 160:11531162

Liu S, Zhang X, Pumphrey MO, Stack RW, Gill BS, Anderson JA (2006) Complex microcolinearity among wheat, rice, and barley revealed by fine mapping of the genomic region harboring a major QTL for resistance to Fusarium head blight in wheat. Funct Integr Genomics 6:83-89

$\mathrm{Lu} \mathrm{H}$, Faris JD (2006) Macro- and microcolinearity between the genomic region of wheat chromosome $5 \mathrm{~B}$ containing the $T_{s n 1}$ gene and the rice genome. Funct Integr Genomics 6:90-103

Mihara M, Itoh T, Izawa T (2008) In silico identification of short nucleotide sequences associated with gene expression of pollen development in rice. Plant Cell Physiol 49:1451-1464

Moore G, Devos KM, Wang Z, Gale MD (1995) Cereal genome evolution. Grasses, line up and form a circle. Curr Biol 5:737739
Olsson AS, Engstrom P, Soderman E (2004) The homeobox genes $A T H B 12$ and $A T H B 7$ encode potential regulators of growth in response to water deficit in Arabidopsis. Plant Mol Biol 55:663677

Ozdemir BS, Hernandez P, Filiz E, Budak H (2008) Brachypodium genomics. Int J Plant Genomics 2008:536104

Palena CM, Gonzalez DH, Chan RL (1999) A monomer-dimer equilibrium modulates the interaction of the sunflower homeodomain leucine-zipper protein Hahb-4 with DNA. Biochem J 341 (Pt 1):81-87

Palena CM, Tron AE, Bertoncini CW, Gonzalez DH, Chan RL (2001) Positively charged residues at the N-terminal arm of the homeodomain are required for efficient DNA binding by homeodomain-leucine zipper proteins. J Mol Biol 308:39-47

Paterson AH, Bowers JE, Chapman BA (2004) Ancient polyploidization predating divergence of the cereals, and its consequences for comparative genomics. Proc Natl Acad Sci USA 101:9903-9908

Paterson AH, Bowers JE, Rm B, Dubchak I, Grimwood J, Gundlach H, Haberer G, Hellsten U, Mitros T, Poliakov A, Schmutz J, Spannagl M, Tang H, Wang X, Wicker T, Bharti AK, Chapman J, Feltus FA, Gowik U, Grigoriev IV, Lyons E, Maher CA, Martis M, Narechania A, Otillar RP, Penning BW, Salamov AA, Wang Y, Zhang L, Carpita NC, Freeling M, Gingle AR, Hash CT, Keller B, Klein P, Kresovich S, McCann MC, Ming R, Peterson DG, Mehboob-ur-Rahman WD, Westhoff P, Mayer KFX, Messing J, Rokhsar DS (2009) The Sorghum bicolor genome and the diversification of grasses. Nature 457:551-556

Pourkheirandish M, Komatsuda T (2007) The importance of barley genetics and domestication in a global perspective. Ann Bot (Lond) 100:999-1008

Pourkheirandish M, Wicker T, Stein N, Fujimura T, Komatsuda T (2007) Analysis of the barley chromosome 2 region containing the six-rowed spike gene vrs 1 reveals a breakdown of rice-barley micro collinearity by a transposition. Theor Appl Genet 114:1357-1365

Rostoks N, Mudie S, Cardle L, Russell J, Ramsay L, Booth A, Svensson JT, Wanamaker SI, Walia H, Rodriguez EM, Hedley PE, Liu H, Morris J, Close TJ, Marshall DF, Waugh R (2005) Genome-wide SNP discovery and linkage analysis in barley based on genes responsive to abiotic stress. Mol Genet Genomics 274:515-527

Rueda EC, Dezar CA, Gonzalez DH, Chan RL (2005) Hahb-10, a sunflower homeobox-leucine zipper gene, is regulated by light quality and quantity, and promotes early flowering when expressed in Arabidopsis. Plant Cell Physiol 46:1954-1963

Sasaki T, Antonio BA (2009) Plant genomics: Sorghum in sequence. Nature 457:547-548

Sessa G, Morelli G, Ruberti I (1993) The Athb-1 and -2 HD-Zip domains homodimerize forming complexes of different DNA binding specificities. EMBO J 12:3507-3517

Shepherd K, Islam A (1981) Wheat: barley hybrids-the first eighty years. In: Evans R, Peacock K (eds) Wheat science today and tomorrow. Cambridge University Press, Cambridge, pp 107-128

Srinivasachary DMM, Gale MD, Devos KM (2007) Comparative analyses reveal high levels of conserved colinearity between the finger millet and rice genomes. Theor Appl Genet 115:489-499

Swigonova Z, Lai J, Ma J, Ramakrishna W, Llaca V, Bennetzen JL, Messing J (2004) On the tetraploid origin of the maize genome. Comp Funct Genomics 5:281-284

Tarchini R, Biddle P, Wineland R, Tingey S, Rafalski A (2000) The complete sequence of $340 \mathrm{~kb}$ of DNA around the rice Adh1-adh2 region reveals interrupted colinearity with maize chromosome 4 . Plant Cell 12:381-391

Tron AE, Welchen E, Gonzalez DH (2004) Engineering the loop region of a homeodomain-leucine zipper protein promotes 
efficient binding to a monomeric DNA binding site. Biochemistry 43:15845-15851

Vegetti A, Anton AM (1995) Some evolution trends in the inflorescence of Poaceae. FLORA 190:225-228

Wei F, Coe E, Nelson W, Bharti AK, Engler F, Butler E, Kim H, Goicoechea JL, Chen M, Lee S, Fuks G, Sanchez-Villeda H, Schroeder S, Fang Z, McMullen M, Davis G, Bowers JE, Paterson AH, Schaeffer M, Gardiner J, Cone K, Messing J, Soderlund C, Wing RA (2007) Physical and genetic structure of the maize genome reflects its complex evolutionary history. PLoS Genet 3:e123
Yamaguchi T, Lee DY, Miyao A, Hirochika H, An G, Hirano HY (2006) Functional diversification of the two C-class MADS box genes OSMADS3 and OSMADS58 in Oryza sativa. Plant Cell 18:15-28

Yan L, Loukoianov A, Tranquilli G, Helguera M, Fahima T, Dubcovsky J (2003) Positional cloning of the wheat vernalization gene VRN1. Proc Natl Acad Sci USA 100:6263-6268

Zanetti ME, Chan RL, Godoy AV, Gonzalez DH, Casalongue CA (2004) Homeodomain-leucine zipper proteins interact with a plant homologue of the transcriptional co-activator multiprotein bridging factor 1. J Biochem Mol Biol 37:320-324 